\title{
Refractory alveolar rhabdomyosarcoma in an 11-year-old male
}

\author{
Cora A. Ricker, ${ }^{1}$ Andrew D. Woods, ${ }^{1}$ William Simonson, ${ }^{2}$ Melvin Lathara, ${ }^{3}$ \\ Ganapati Srinivasa, ${ }^{3}$ Erin R. Rudzinski, ${ }^{4}$ Atiya Mansoor, ${ }^{1}$ Robert G. Irwin, ${ }^{2}$ \\ Charles Keller, ${ }^{1}$ and Noah E. Berlow ${ }^{1}$ \\ ${ }^{1}$ Children's Cancer Therapy Development Institute, Beaverton, Oregon 97005, USA; ${ }^{2}$ Mary Bridge Hospital, \\ Tacoma, Washington 98403, USA; ${ }^{3}$ Omics Data Automation, Beaverton, Oregon 97005, USA; ${ }^{4}$ Department of \\ Laboratories, Seattle Children's Hospital, Seattle, Washington 98105, USA
}

\begin{abstract}
Rhabdomyosarcoma (RMS) is a mesenchymal malignancy phenocopying muscle and is among the leading causes of death from childhood cancer. Metastatic alveolar rhabdomyosarcoma is the most aggressive subtype with an $8 \% 5$-yr disease-free survival rate when a chromosomal fusion is present and a $29 \% 5$-yr disease-free survival rate when negative for a fusion event. The underlying biology of $P A X$-fusion-negative alveolar rhabdomyosarcoma remains largely unexplored and is exceedingly rare in Li-Fraumeni syndrome patients. Here, we present the case of an 11-yr-old male with fusion-negative alveolar rhabdomyosarcoma studied at end of life with a comprehensive functional genomics characterization, resulting in identification of potential therapeutic targets for broader investigation.
\end{abstract}

[Supplemental material is available for this article.]

\section{INTRODUCTION}

Corresponding authors: noah@cc-tdi.org; charles@cc-tdi.org

(c) 2021 Ricker et al. This article is distributed under the terms of the Creative Commons Attribution-NonCommercial License, which permits reuse and redistribution, except for commercial purposes, provided that the original author and source are credited.

Ontology term:

rhabdomyosarcoma

Published by Cold Spring Harbor Laboratory Press

doi:10.1101/mcs.a005983
Rhabdomyosarcoma (RMS) is the most common soft-tissue sarcoma occurring in children with two major subtypes, embryonal and alveolar (Rudzinski et al. 2017). Embryonal rhabdomyosarcoma (ERMS) tends to have a more favorable prognosis and is mainly seen in the orbit, head, neck, and retroperitoneum (Rudzinski et al. 2017). Alveolar rhabdomyosarcoma (ARMS), on the other hand, has a less favorable prognosis and often involves the trunk and extremities (Rudzinski et al. 2017). Although PAX3:FOXO1 or PAX7:FOXO1 chimeric oncogenes are found in the majority of ARMS cases, $15 \%$ of rhabdomyosarcomas with available fusion data are negative for FOXO1 rearrangement (Rudzinski et al. 2017). These cases are prognostically similar to embryonal rhabdomyosarcoma, which dictates their risk-based treatment (Rudzinski et al. 2017). In general, fusion status serves as a better predictor of prognosis than histology (Rudzinski et al. 2017). The 5-yr event-free survival (EFS) for fusion-negative ARMS is $29 \%$ (Rudzinski et al. 2017). Despite advancements in uncovering the clinical and mutational profiles of the myriad RMS subtypes, such as recurrent TP53 tumor suppressor gene loss of function in PAX fusion-negative (PFN) RMS, survival rates have not seen similar significant improvement (Breneman et al. 2003; Williams et al. 2004; Davis and Keller 2012; Malempati and Hawkins 2012; Rudzinski et al. 2017).

Several predisposition syndromes such as neurofibromatosis, Costello syndrome, and Li-Fraumeni syndrome have been linked to sarcomas (Farid and Ngeow 2016). Li-Fraumeni syndrome (LFS) is an autosomal dominant disorder commonly characterized by heterozygous germline mutations in TP53. LFS predisposes patients to a wide range of 
malignancies, including sarcomas, which account for $25 \%-35 \%$ of all LFS-associated tumors and 1\%-10\% of RMS (Olivier et al. 2003; Gonzalez et al. 2009; Palmero et al. 2010; Ognjanovic et al. 2012). Data suggest that overall survival outcomes were improved with early detection of tumors through surveillance of patients with TP53 mutations (Ballinger et al. 2015). However, detection of LFS can be challenging for clinicians. Presentation of clinical characteristics, surveillance strategies, and therapeutic interventions can all differ between patients given the wide range of malignancies associated with LFS. Most TP53 mutations associated with LFS are missense mutations, which may or may not significantly affect protein function (Olivier et al. 2003). The ambiguity of classifying these mutations as benign or deleterious can make clinicians reluctant to diagnose patients with LFS given the psychological ramifications that can come with diagnosis and active surveillance thereafter. The International Agency for Research on Cancer (IARC) TP53 Database has compiled TP53 mutations reported in published literature and can be used for further risk assessment (Hainaut et al. 1997). In the context of rhabdomyosarcoma, TP53 mutations have been associated with increased risk of second neoplastic malignancy (Pondrom et al. 2020) and associated with worsened overall survival (Casey et al. 2020), suggesting TP53 plays an important role in clinical progression and TP53 status should be investigated in the course of clinical decision making.

Advanced stage or metastatic patients often receive intensified treatments, which historically show a 3\%-4\% mortality rate from treatment-related toxicities (Crist et al. 2001). Molecularly targeted therapies provide a promising approach for treatment of RMS patients as these therapies have the potential to reduce drug-induced toxicities compared to traditional chemotherapies. Here, we present a case of an 11-yr-old male with fusion-negative ARMS who underwent standard of care followed by exhaustive precision medicine approaches to his progressive disease and a functional genomics characterization at end of life. Drug screening in conjunction with genetic analysis has provided insight to the biological status of the index case and potentially promising drug candidates for future preclinical and clinical studies.

\section{RESULTS}

\section{Clinical Presentation}

An 11-yr-old Caucasian male presented with a tender left upper arm mass. No recent injury was noted, but the patient had a history of prior supracondylar fracture of the distal humerus. Approximately 3 wk later, a magnetic resonance imaging (MRI) of the humerus revealed a lesion within the left upper arm measuring $\sim 2.6 \times 2 \mathrm{~cm}$ in the axial plane and $\sim 3.5 \mathrm{~cm}$ in the craniocaudal dimension within the triceps region of the left upper arm, possibly arising from the medial head of the triceps, with edema and enhancement down to the attachment of the mid-humeral shaft (Fig. 1). The differential diagnosis included soft-tissue fibroma, myxomatous tumor, or neuromuscular mass. Soft-tissue sarcoma was not excluded. Two weeks after presentation, a follow-up X ray of the upper arm mass found that the humerus appeared to be normal without focal bony abnormality, cortical disruption, or periostitis (Supplemental Figs. 1 and 2). Prominent soft tissue was found along the medial aspect of the distal portion of the upper arm corresponding in location to the known mass. The mass was also slightly seen dorsally on the internally rotated view. No calcifications or ossifications were identified in the soft tissues.

Four weeks after presentation, the mass had grown dramatically in size with increased pain. A biopsy of the primary left upper, posterior arm mass revealed a small round blue cell malignancy (Fig. 2). Histology was described as a poorly differentiated malignant neoplasm arranged in loosely cohesive sheets of primitive round to polygonal cells with 

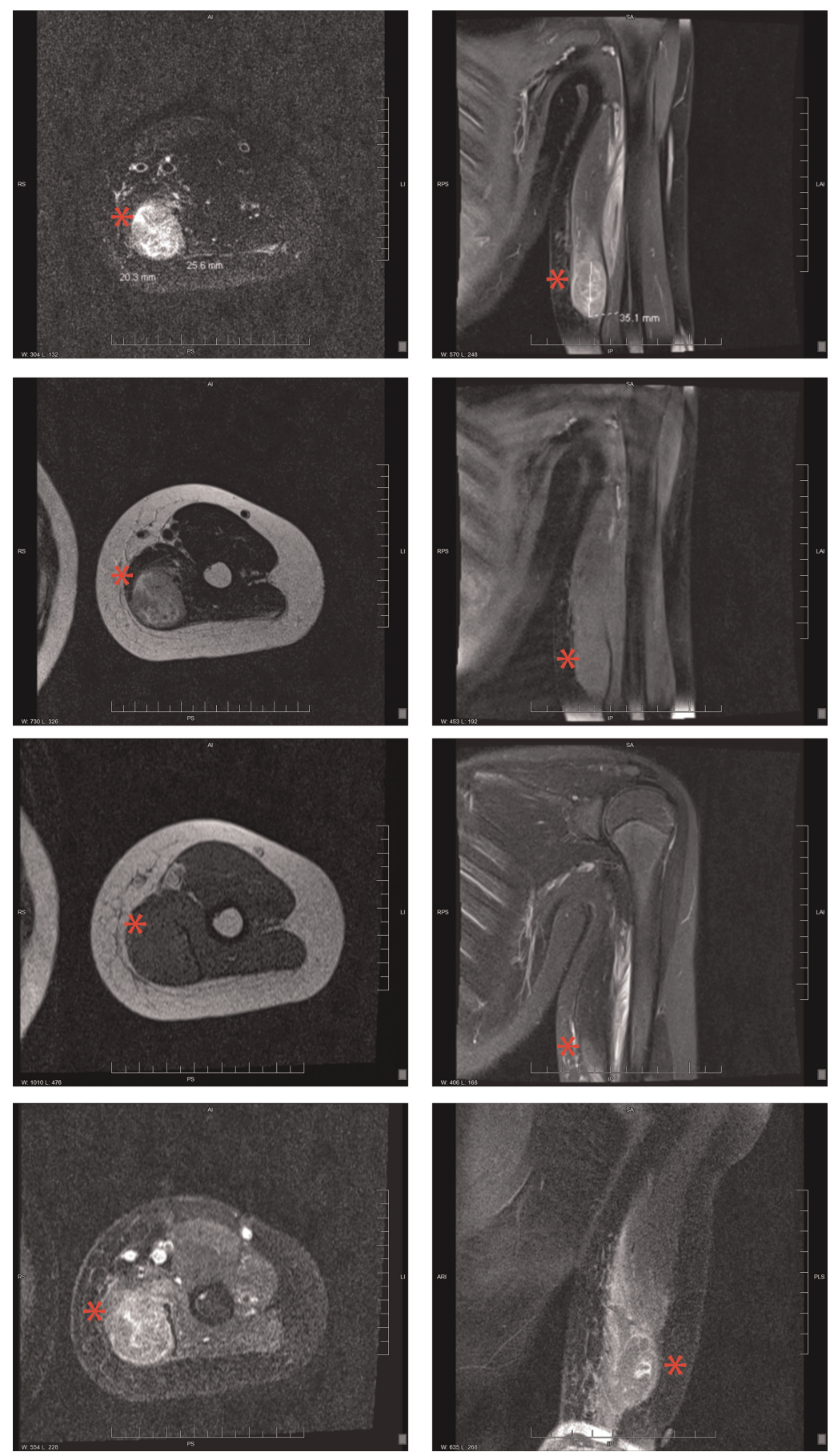

Figure 1. Orthopedic MRI of the humerus. An ovoid lesion (red asterisk) was found within the triceps region of the left upper arm utilizing a 1.5-Tesla magnet with the following sequences: axial $T_{2}$ turbo spin echo (TSE) with fat saturation, axial $\mathrm{T}_{2}$ TSE pulse, axial $\mathrm{T}_{1}$ combination, and axial $\mathrm{T}_{1} \mathrm{TSE}$ after $4.6 \mathrm{~mL}$ of gadolinium $(+\mathrm{C})$ (from top to bottom, left). The following sequences were also obtained: coronal $T_{1}$ TSE $+C$ with fat saturation, coronal $T_{1}$ TSE with fat saturation, coronal short-TI inversion recovery (STIR) combination, and sagittal $T_{1}$ TSE $+C$ with fat saturation (from top to bottom, right). The lesion measured $\sim 2.6 \times 2 \mathrm{~cm}$ in the axial plane and $\sim 3.5 \mathrm{~cm}$ in the craniocaudal dimension (top, left and top, right). The lesion demonstrated $T_{1}$ hypointensity and heterogeneous, but $\mathrm{T}_{2}$ hyperintensity without significant fat saturation. Within the ovoid region, there are two punctate foci of STIR hypointensity (29/19) and $T_{1}$ hypointensity. 

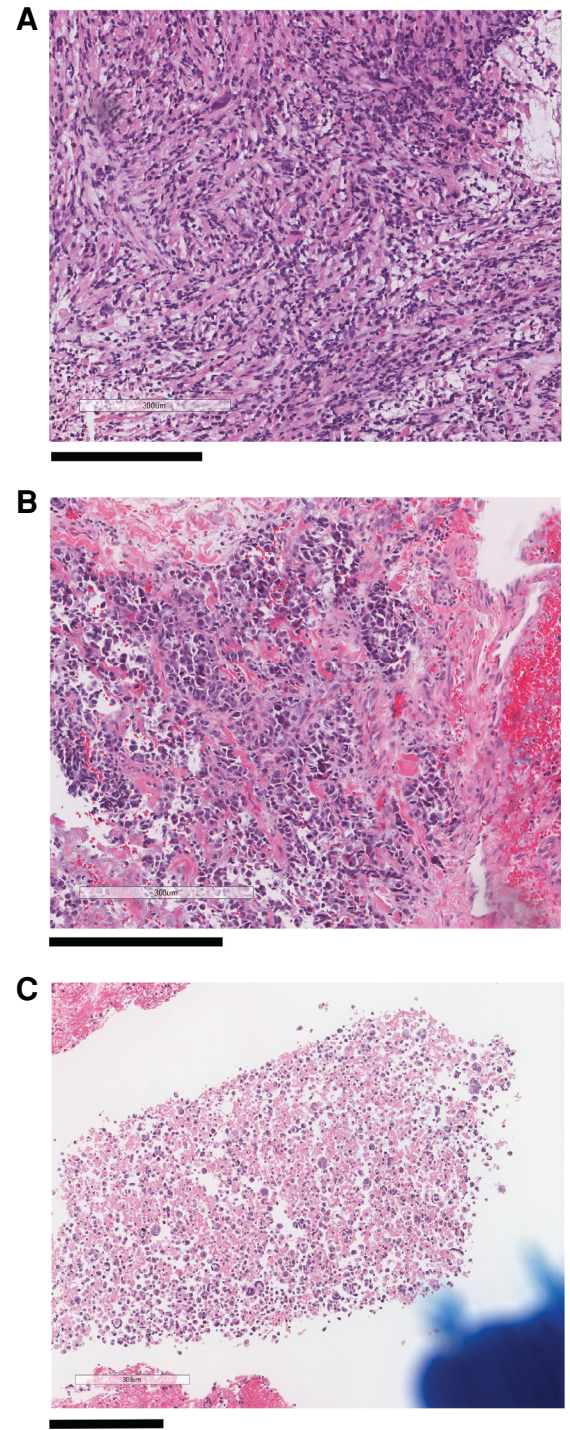

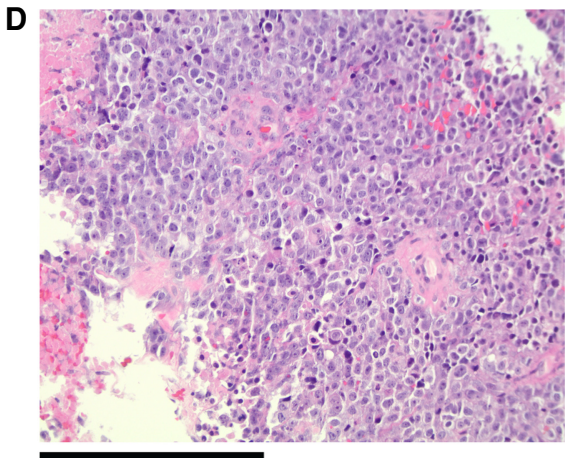

E

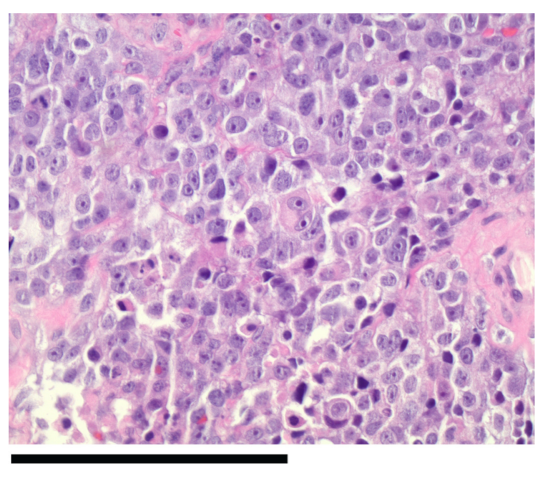

$\mathbf{F}$

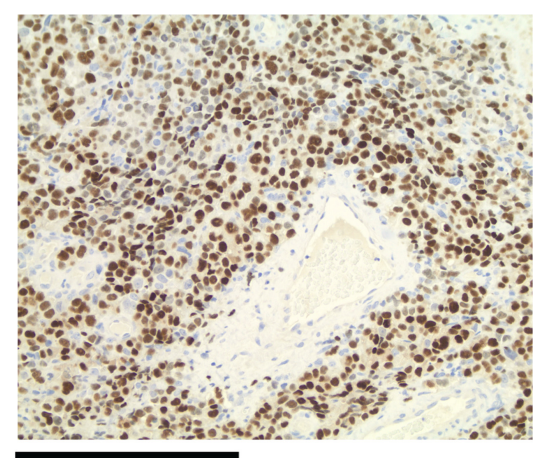

Figure 2. Histology slides from alveolar rhabdomyosarcoma case. Hematoxylin and eosin (H\&E) staining of the left forearm $(A)$, right lower lobe of the lung $(B)$, pleural fluid $(C)$ showing a metastatic small round blue

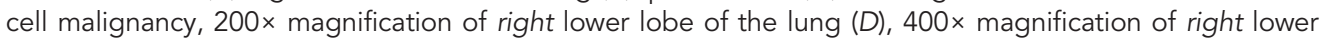
lobe of the lung $(E)$, and $200 \times$ magnification of myogenin staining $(F)$. Scale bars, $300 \mu \mathrm{M}$.

interruptions by few fibrous septa and scattered large vessels. Desmin was reported to be diffusively positive, tumor cell myogenin was positive ( 60\%), and cytokeratin was found negative. PTEN, BAF47 (INI1/SMARCB1), and BRG1 (SMARCA4) were positive. Focal areas of lower cellular cohesion created a vague papillary appearance. The individual tumor cells were reported to be pleomorphic, ranging from intermediate to large. The majority of cells had scant cytoplasm; however, rare cells had a moderate amount of densely eosinophilic cytoplasm with possible cross-striations (rhabdomyoblasts). Multiple scattered multinucleated giant cells were present which also contained deeply eosinophilic cytoplasm. Random cells had enlarged hyperchromatic and anaplastic nuclei. Numerous apoptoses and mitoses were seen. Rare, atypical mitotic figures were noted. The patient was diagnosed with a malignant 
neoplasm of connective and other soft tissue of the upper limb, including shoulder. Tumor morphology suggested an ARMS tumor. However, fluorescence in situ hybridization (FISH) studies were negative for the classic FOXO1 $\mathrm{t}(2,13)$ or $\mathrm{t}(1: 13)$ rearrangements and did not support a diagnosis of ARMS. Bilateral bone marrow biopsies were performed, and were negative for small round blue cell morphology.

Five days after the biopsy, a follow-up postresection multiplanar multisequential MRI scan of the left humerus was performed with and without intravenous contrast. The MRI revealed a heterogeneous mass much larger than the previous scan, measuring $5.2 \times 6 \times 16.8$ $\mathrm{cm}(\mathrm{AP} \times \mathrm{TR} \times \mathrm{CC})$. At the more inferior aspect of the mass, a central area of low signal intensity was found on the T1 weight sequence with peripheral rim enhancement measuring $8 \times$ $2.4 \mathrm{~cm}$ that might represent a central cystic component (possibly representing central necrosis or fluid collection given the recent surgery). The mass abutted the ulnar neurovascular bundle. Nonspecific, small left axillary lymph nodes were noted. Staging investigations through a chest computed tomography (CT) scan revealed no lung metastasis.

Twelve days after diagnosis, the lymph nodes were biopsied and sent for histological analysis. The patient was found to have a metastatic neoplasm consistent with rhabdomyosarcoma in 4 out of 31 lymph nodes. The patient was classified as having stage III, intermediate risk group III RMS, N1, M0 according to the Children's Oncology Group (COG) protocol. The patient began treatment with protocol ARST0531 consisting of 42 wk of vincristine, adriamycin, cyclophosphamide (VAC) and alternating with vincristine and irinotecan (VI). Radiation was started $6 \mathrm{wk}$ after diagnosis and given for a month and a half for local control (5040 cGy in 28fx at 180cGy/fx using 3DCRT). Complications of treatment included mucositis, Clostridioides difficile infection, headache, and exacerbated neuropathy.

Sixteen months after diagnosis (6 mo after chemotherapy was completed), chest CT showed new lung masses, raising concerns of metastatic recurrence. No arm pain was noted. On the positron emission tomography (PET)-CT scan, the fluorine-18-deoxyglucose (FDG) signal in the right lower lung lobe mass was most consistent with progressive metastatic disease. A new left lower lung lobe nodule further raised concern of metastatic disease, despite lack of discernable FDG activity in the left lower lobe. Lobectomy of the right lower lobe revealed a small round blue cell tumor, consistent with metastatic rhabdomyosarcoma $(1.6 \mathrm{~cm})$ with vascular invasion and positive bronchovascular margin. Clear margins were not obtained. The patient was diagnosed with relapsed stage IV ARMS.

Eighteen and a half months after diagnosis (two and a half months after relapse), the patient began reinduction chemotherapy with vinorelbine, cyclophosphamide, and temsirolimus (ARST-0921). Independent of ARST-0921, the patient also supplemented with glutamine and vitamin $B_{2}$, as well as $C B D / T H C$ to control pain and anxiety. There was no overlap in supplement regimens. Three months after starting reinduction chemotherapy, the patient completed $28 \mathrm{~d}$ of radiation to the right lung. Relapse treatment was given over a period of $7.5 \mathrm{mo}$. At the end of relapse treatment, a full body MRI and local CT showed stable disease. Possible nodules in the left lung and a mass in the lower left forearm were noted.

Two months after completing relapse treatment ( $28 \mathrm{mo}$ after initial diagnosis), the patient enrolled in clinical trial ADVL1515 and started treatment with prexasertib, a CHEK1 inhibitor. Radiographic scans one month after enrollment in ADVL1515 showed new cancerous growth in the arms and lungs. Prexasertib treatment was stopped and the patient enrolled in clinical trial ADVL1312 and began treatment with MK-1775, a WEE1 inhibitor. After one month of MK-1775 treatment, radiographic scans showed continued progression, and therapy was stopped. The patient had also enrolled in the Pediatric MATCH trial (APEC1621), but no targetable mutations were found from genomic sequencing.

Thirty months after initial diagnosis the patient began daily oral pazopanib, a 5-d course of temozolomide, and radiotherapy to the left forearm and lungs for local control. A biopsy 
was performed for clinical trial eligibility determination and development of a patient-derived xenograft at Champions Oncology. One month later (31 mo after initial diagnosis) the patient was determined to be eligible for clinical trial NCT-02162732, a genomically guided therapy trial for children with cancer. The patient began treatment with vorinostat and palbociclib and supplemented with myrrh oil. The patient was also treated with weekly thoracentesis.

Two weeks later, the patient presented to the ER with shortness of breath and chest pain. The necrotic tumor in the left arm was opened and debrided and the patient received i.v. clindamycin. A drain was placed for the patient's malignant pleural effusion with loculation. A week later (32 mo after initial diagnosis), the patient reported a moderate increase in symptoms attributed to his disease over a 24-h period. Doppler ultrasound showed acute occlusive venous thrombosis involving the right internal jugular vein, right subclavian vein, right axillary vein, and right cephalic vein, with additional nonocclusive thrombus in the right basilic vein (superior vena cava syndrome syndrome). CT chest scans showed near complete opacification of the right hemithorax (presumed malignant), extensive thrombosis, and an increased left lower lobe lesion measuring $3.9 \mathrm{~cm}$. Pulmonary fluid increased with drainage reaching $\sim 1.5 \mathrm{~L} /$ day, and the patient required increased support and spent more time on a bilevel positive airway pressure (BiPAP) machine. Medical treatment was not felt to be a viable option given the patient's significant bleeding risk. Vorinostat and palbociclib, as well as supportive care, were discontinued by parental request. The patient was taken off BiPAP and passed away at the age of 14, 33 mo after initial diagnosis. The discharge diagnosis was progressive hypoxic respiratory failure, malignant pleural effusion, and relapsed rhabdomyosarcoma.

\section{Genomic Analysis}

Cytogenetics revealed 46,XY[20], normal male karyotype. Previous testing by OncoPlex identified a TP53 p.V172F (Tier 2A) mutation with associated loss of heterozygosity, suggesting that both copies of the gene are expected to be inactivated in the tumor tissue. FGFR1 amplification (Tier $2 \mathrm{~A} / 4 \mathrm{~A}, \log _{2}$ tumor/normal read ratio $=0.96$ by whole-exome sequencing [WES]) and MYCL amplification (Tier $5 \mathrm{~A}, \log _{2}$ tumor/normal read ratio $=1.91$ by WES) were both present. Targeted sequencing (Ambry Genetics) confirmed that the patient shared the same TP53 mutation (V172F) as his mother. Two previous generations also had early-onset breast cancer on the mother's side (maternal grandmother and great grandmother).

To identify further genomic alterations and potential therapeutic targets, we sequenced and analyzed DNA and RNA isolated from a tumor tissue sample from the left arm, as well as DNA from patient-matched normal tissue. Tissue for sequencing experiments were provided postmortem. After filtering for nonsynonymous mutations not identified in the database of single-nucleotide polymorphisms (Sherry et al. 2001), 109 mutations were found. No somatic mutations were found in genes commonly mutated in PFN tumors, including TP53, HRAS, KRAS, NRAS, FGFR4, PIK3CA, NF1, FBXW7, and BCOR (Stratton et al. 1989; Taylor et al. 2000, 2009; Shern et al. 2014). However, two germline mutations were found in TP53, including the same variant found in the patient's mother (c.514G > T p.Val172Phe) (Table 1), which is considered likely pathogenic for hereditary cancer-predisposing syndrome (ClinVar ID: 428909). Another missense variant in TP53 (c.215C > G p.Pro72Arg) was found to alter drug response in several neoplasms but found to not be associated with Li-Fraumeni syndrome 1 (ClinVar ID: 12351). TP53 mutations have previously been associated with response to the majority (six of eight) therapeutic regimens administered to the patient (Table 2). No somatic mutations were found in genes previously reported to be recurrently mutated in RMS (Shern et al. 2014); however, germline protein coding mutations were found in 


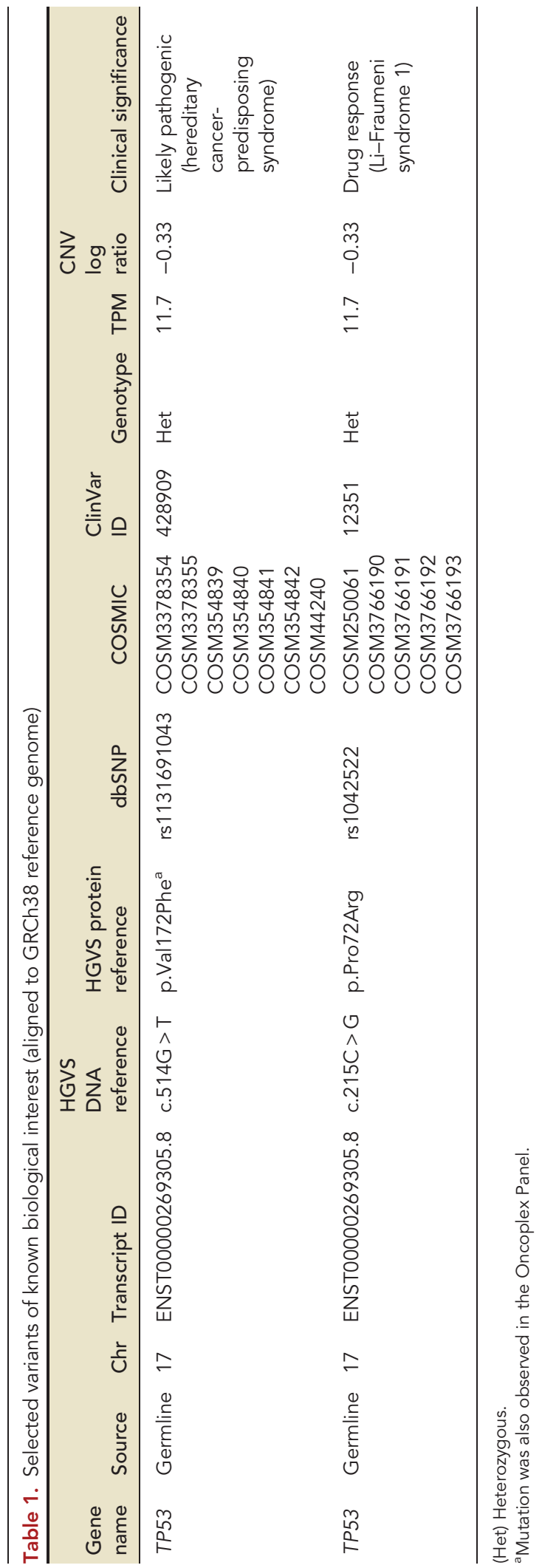


Table 2. Possible treatment-related genomic features

\begin{tabular}{|c|c|c|c|}
\hline $\begin{array}{l}\text { Protocol/clinical } \\
\text { trial }\end{array}$ & Therapeutic regimen & Molecular target & Genomic features \\
\hline ARST0531 & $\begin{array}{l}\text { VAC (vincristine, adriamycin, } \\
\text { cyclophosphamide) + alternating } \\
\text { vincristine and irinotecan (VI) }\end{array}$ & N/A & $\begin{array}{l}\text { Cyclophosphamide (TP53: PMID17388661, } \\
\text { PMID16243804, PMID26438783), irinotecan } \\
\text { (TP53, PMID25567130) }\end{array}$ \\
\hline ARST091 & $\begin{array}{l}\text { Vinorelbine, cyclophosphamide, } \\
\text { temsirolimus }\end{array}$ & mTOR & $\begin{array}{l}\text { Cyclophosphamide (TP53: PMID17388661, } \\
\text { PMID16243804, PMID26438783) }\end{array}$ \\
\hline ADVL1515 & Prexasertib & CHEK1 & $\mathrm{N} / \mathrm{A}$ \\
\hline ADVL1312 & MK-1775 & WEE1 & $\begin{array}{l}\text { TP53: PMID27601554, PMID27196784, } \\
\text { PMID21992793 }\end{array}$ \\
\hline ADVL1312 & Pazopanib & $\begin{array}{l}\text { Tyrosine kinases, } \\
\text { VEGF }\end{array}$ & TP53: PMID26646755, PMID25669829 \\
\hline ADVL1312 & Temozolomide & Alkylating agent & TP53: PMID21730979, PMID24248532 \\
\hline NCT02162732 & Vorinostat & $\begin{array}{l}\text { HDAC inhibitor } \\
\text { (class I and } \\
\text { class II) }\end{array}$ & TP53: PMID26009011, PMID25669829 \\
\hline NCT02162732 & $\begin{array}{l}\text { Palbociclib (supplemented with myrrh oil); } \\
\text { weekly thoracentesis }\end{array}$ & $\begin{array}{l}\text { CDK4/CDK6 } \\
\text { inhibitor }\end{array}$ & $\mathrm{N} / \mathrm{A}$ \\
\hline
\end{tabular}

FGFR4 (c.28G > A p.Val10lle; c.407C > T p.Pro136Leu) and nonsense mediated decay variants were found in NF1 (c.37T > C p.Ter13Arg).

In addition to the germline variants found in TP53, a germline disruptive in-frame insertion variant was found in FAM83D (c.345 347dupGGC p.Ala116dup), along with copy-number gain in FAM83D ( $\log _{2}$ ratio 0.7). Further, FAM83D is overexpressed (23.5-fold, 2.84 TPM vs. 0.1207 TPM) compared to median expression of the GTEx normal skeletal muscle tissue cohort $(n=803$ ). The clinical significance of the FAM83D mutation (c.345 347dupGGC p.Ala116dup) is at present unknown; however, FAM83D overexpression occurs frequently in hepatocellular carcinoma, ovarian cancer, and metastatic lung adenocarcinomas (Inamura et al. 2007; Ramakrishna et al. 2010; Liao et al. 2015; Wang et al. 2015) and correlated with significantly decreased overall survival and metastatic relapse-free survival in breast cancer (Wang et al. 2013). Further, elevated FAM83D was found to correlate with TP53 mutations and genome instability (Walian et al. 2016).

Other somatic mutations were identified, including KDM2B and HCAR1. Both had truncating mutations and copy-number loss ( $\log _{2}$ ratio $\left.<-0.4\right)$. Several genes located on Chromosome 1 were found to be mutated, amplified, and have transcripts per million (TPM) of $>100$ or $<10$ (Fig. 3). Using RNA isolated from the tumor of the left arm, 10 gene fusions were identified using the STAR-Fusion analysis pipeline, with many fused genes located on Chromosome 1 (Fig. 3; Supplemental Table 1). Notably, RNA sequencing data from the tumor sample procured following recurrence did not identify any of the typical gene fusion events associated with alveolar rhabdomyosarcoma, including PAX3:FOXO1, PAX7: FOXO1, PAX3:NCOA1, PAX3:NCOA2, and PAX3:FOXO4. A second gene fusion analysis pipeline FusionCatcher also did not find evidence to support the presence of the ARMS-associated gene fusions (Supplemental Table 2). The lack of evidence of PAX3:FOXO1 in the patient's tumor at diagnosis (confirmed by FISH analysis) and lack of any evidence to support any ARMS-associated fusion in the recurrent tumor suggest the patient was unlikely to bear typical ARMS-associated gene fusions throughout the course of disease.

The presence of clustered fusion events and 10 switches in copy-number status suggests Chromosome 1 may be chromothripsis-positive with high confidence event on 


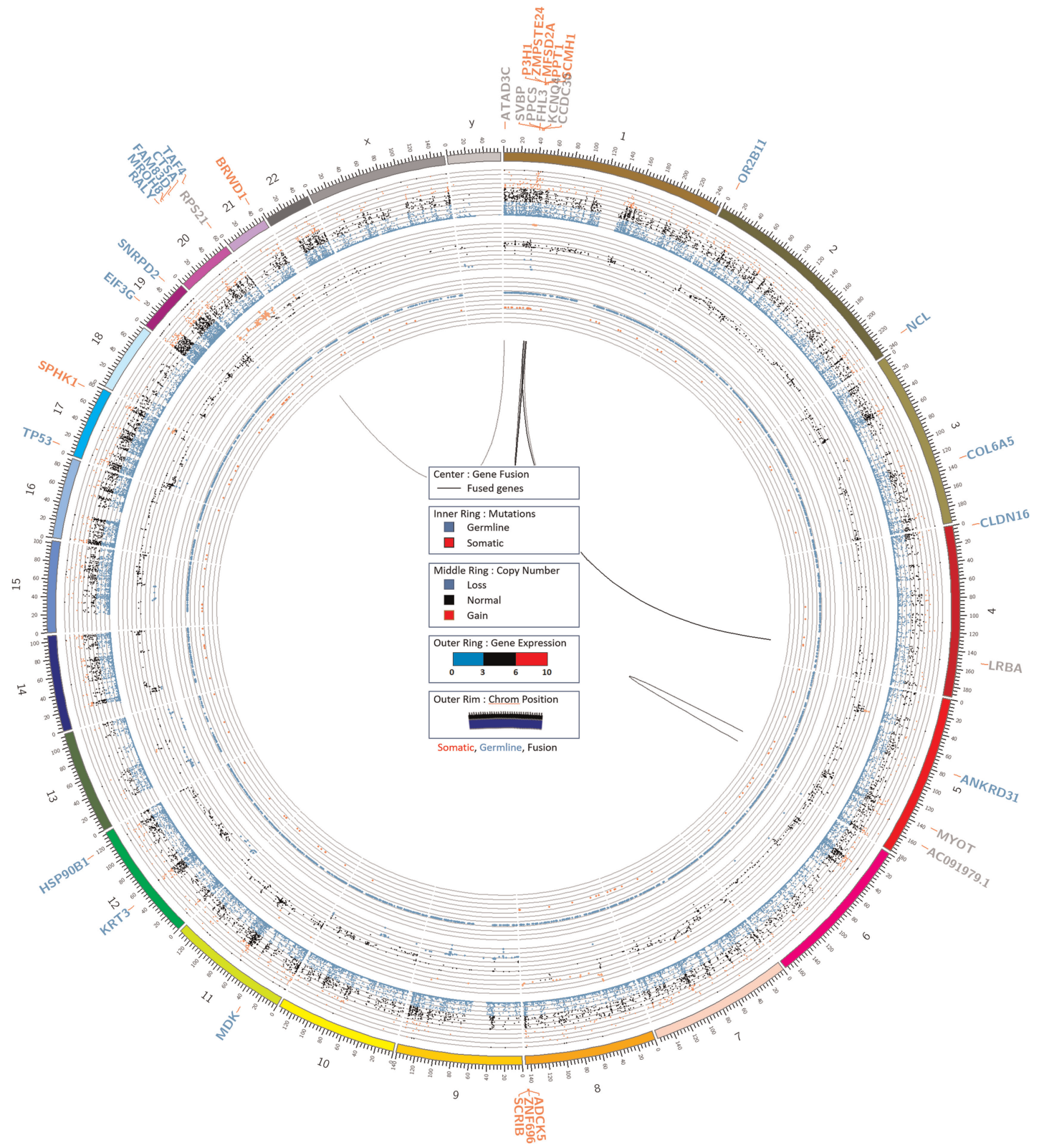

Figure 3. Circos plot. A Circos plot was generated using tumor DNA exome, normal DNA exome, and tumor RNA sequencing data. Exome data was analyzed for somatic point mutation, indel, and copy-number variation data, as denoted by the inner and middle rings, respectively. Gene expression from RNA sequencing data was plotted on the outer ring. Genes listed in blue bear germline mutations, whereas genes listed in red bear somatic mutations.

Chromosome 1 (Bolkestein et al. 2020; Cortés-Ciriano et al. 2020). Notably, the lesions appear over a small region of the genome, suggesting the clustered variants may be classified as a kataegis event. FGFR4, CCND2, and IGF2 were found to be the top genes overexpressed in PFN RMS cases in a previous study (Shern et al. 2014) and were also found to be overexpressed (12-fold, 16-fold, and 153-fold overexpression, respectively) in the 
patient's tumor compared to a panel of normal skeletal muscle tissue samples $(n=803)$ from the Genotype-Tissue Expression (GTEx) project (Consortium 2013). A complete list of gene mutations and associated gene expression and copy-number variation data is provided in Supplemental Table 3 and Supplemental Table 4.

Given the relative rarity of fusion-negative ARMS cases among the RMS clinical milieu, we investigated genomic similarities between the index case and other clinical RMS samples using a previously published hierarchical clustering dendrogram approach (Supplemental Fig. 3; Ricker et al. 2020). Similar to previous findings regarding genomic similarities between ERMS and fusion-negative ARMS (Williamson et al. 2010), CF-00449 clusters among ERMS samples, as do the majority of other fusion-negative ARMS samples. Taken together, the histopathology designation of ARMS by multiple pathologists, the lack of evidence of fusions from FISH analysis and RNA sequencing analysis, and the genomic similarity to ERMS more so than ARMS strongly support the diagnosis of CF-00449 as a fusion-negative ARMS.

\section{Functional Analyses}

To identify molecular compounds that would inhibit tumor growth, we conducted a chemical screen consisting of a panel of 60 agents from a clinically relevant drug portfolio on cells harvested from the patient's pleural fluid (Table 3; Supplemental Fig. 4). Several drugs were highly active, including panobinostat $\left(\mathrm{IC}_{50}=6 \mathrm{nM}\right.$, pan HDAC inhibitor), brefeldin $\mathrm{A}\left(\mathrm{IC}_{50}=21 \mathrm{nM}\right.$, ATPase inhibitor), CUDC-907 (IC $\mathrm{C}_{50}=99 \mathrm{nM}, \mathrm{HDAC} 1 / 2 / 3 / 10+\mathrm{PI}$ KKa inhibitor), YM155 (IC $50=9 \mathrm{nM}$, Survivin inhibitor), INK128 (IC $50=5 \mathrm{nM}, \mathrm{mTORC} 1 / 2), \mathrm{MK}-1775$ $\left(I C_{50}=79 \mathrm{nM}\right.$, WEE1), thapsigargin $\left(I C_{50}=9 \mathrm{nM}, \mathrm{SERCA}\right), \mathrm{BMS}-754807\left(\mathrm{IC}_{50}=66 \mathrm{nM}\right.$, IGF1R + AURK inhibitor), JQ1(IC $C_{50}=92 \mathrm{nM}, \mathrm{BET}$ (BRD4) inhibitor), and mithramycin A $\left(\mathrm{IC}_{50}=89 \mathrm{nM}, \mathrm{SP} 1\right.$ inhibitor). TP53 mutations were associated with response in multiple high and moderate sensitivity agents (Table 4). Chemical screening was performed using cells isolated pleural effusion fluid while the patient was still alive, and did not influence clinical decision making.

The patient received the nonselective class I/II histone deacetylase (HDAC) inhibitor, vorinostat, with limited response. Although HDAC inhibitors panobinostat, CUDC-907, and entinostat were found to impair growth of tumor cells in vitro, the HDAC inhibitor CUDC101 did not demonstrate significant activity in vitro. Panobinostat is a pan-HDAC inhibitor with activity against all HDAC enzymes, whereas CUDC-907 inhibits class I-III HDAC genes plus phosphoinositide 3-kinase a (PI3K- $\alpha$ ), and entinostat inhibits classes I and III. Meanwhile, CUDC-101 inhibits class I/II HDACs as well as epidermal growth factor receptor (EGFR) and human epidermal growth factor receptor (HER). The discrepancy of response to tested HDAC inhibitors can potentially be explained by differences in their mechanism of action (Li and Seto 2016). These results suggest that inhibition of class I and II HDACs may not be sufficient for tumor suppression of PFN ARMS and targeting of other HDAC classes may have a more significant contribution to tumor survival. Further supporting this notion, panobinostat and entinostat were found to have different consequences in PAX3:FOXO1 ARMS (Bharathy et al. 2018).

The WEE1 inhibitor, MK-1775, which had high potency in vitro $\left(\mathrm{IC}_{50}=79 \mathrm{nM}\right)$, was administered to the patient under trial ADVL1312 and did not prevent progression. Prexasertib (LY2606368) was also used to treat the patient under trial ADVL1515 but did not suppress the patient's tumor growth, consistent with the limited in vitro activity of prexasertib $\left(I C_{50}>10 \mu \mathrm{M}\right)$.

Clinically, temsirolimus (mTOR inhibitor) in combination with vinorelbine and cyclophosphamide resulted in stable disease with potential tumor growth. INK128 (mTORC1/ mTORC2 inhibitor) and BKM120 (IC $\mathrm{C}_{50}=198 \mathrm{nM}$, mTOR and PI3K inhibitor) both had high activity in tumor cells, whereas sirolimus (also an mTOR inhibitor) did not have activity in 


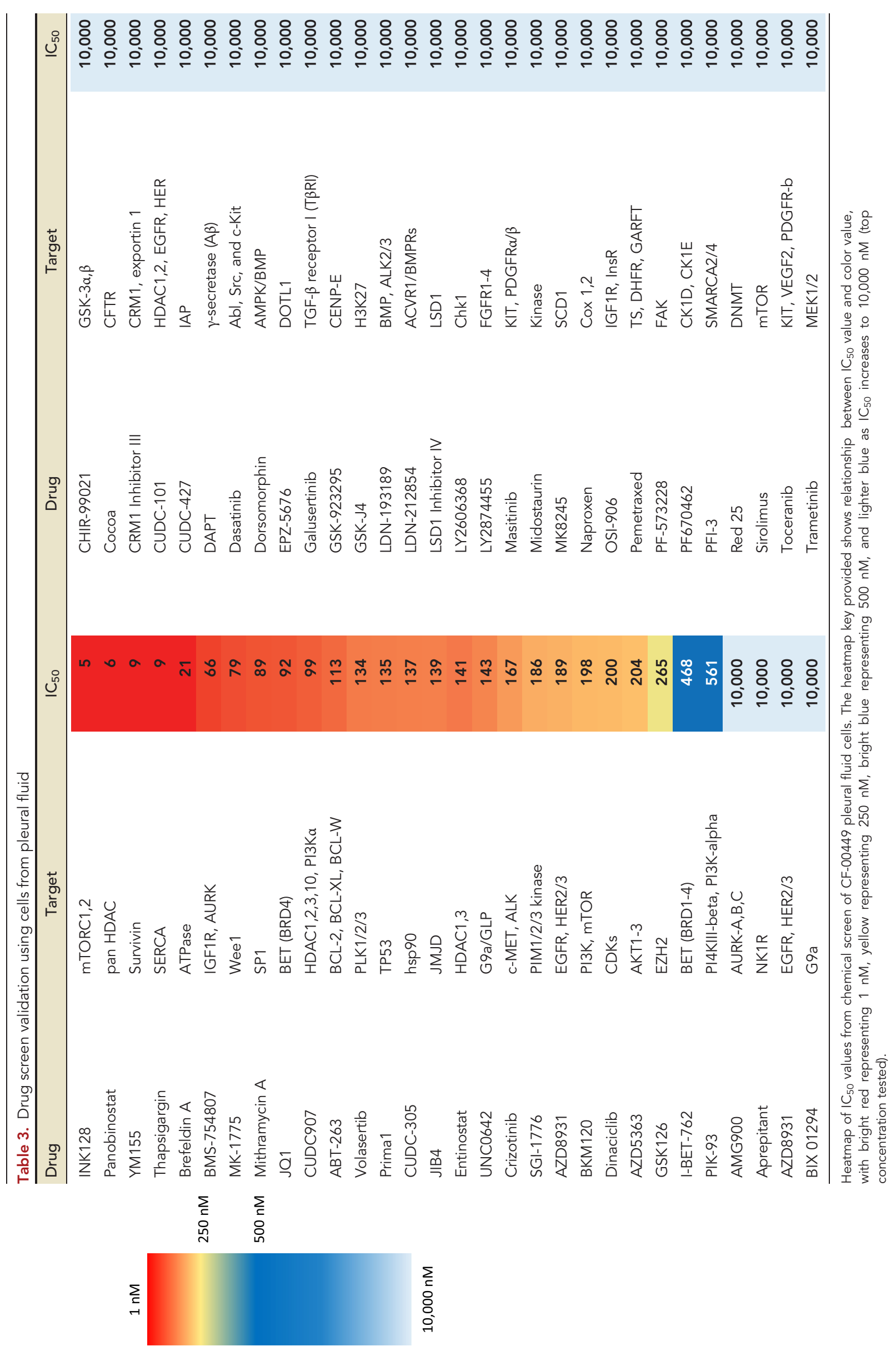




\begin{tabular}{|c|c|c|c|}
\hline & Drug & Inhibitor & $\begin{array}{l}\text { Genomic-related } \\
\text { features }\end{array}$ \\
\hline \multirow[t]{10}{*}{ High activity } & Panobinostat & pan HDAC & N/A \\
\hline & Brefeldin A & ATPase & $\mathrm{N} / \mathrm{A}$ \\
\hline & CUDC-907 & $\operatorname{HDAC} 1,2,3,10, \mathrm{PI} 3 \mathrm{Ka}$ & N/A \\
\hline & YM155 & Survivin inhibitor & N/A \\
\hline & INK128 & $\mathrm{mTORC} 1 / 2$ & $\mathrm{~N} / \mathrm{A}$ \\
\hline & MK-1775 & WEE1 & $\begin{array}{l}\text { TP53: PMID27601554, } \\
\text { PMID27196784, } \\
\text { PMID21992793 }\end{array}$ \\
\hline & Thapsigargin & SERCA & N/A \\
\hline & BMS-754807 & IGF1R, AURK & IGF2: PMID19996272 \\
\hline & JQ1 & BET (BRD4) & N/A \\
\hline & Mithramycin A & SP1 & N/A \\
\hline \multirow[t]{15}{*}{ Moderate activity } & GSK126 & $\mathrm{EZH} 2$ & $\mathrm{~N} / \mathrm{A}$ \\
\hline & CUDC-305 & HSP90 & N/A \\
\hline & AZD5363 & AKT1-3 & $\begin{array}{l}\text { TP53: PMID24694055, } \\
\text { PMID17145876, } \\
\text { PMID17455259 }\end{array}$ \\
\hline & ABT-263 & BCL-2, BCL-XL, BCL-W & TP53: PMID24694055 \\
\hline & I-BET-762 (BET) & BRD1-4 & $\mathrm{N} / \mathrm{A}$ \\
\hline & Dinaciclib & CDKs & N/A \\
\hline & Entinostat & $\operatorname{HDAC} 1,3$ & $\mathrm{~N} / \mathrm{A}$ \\
\hline & Crizotinib & c-MET, ALK & TP53: PMID24694055 \\
\hline & BKM120 & $\mathrm{PI} 3 \mathrm{~K}, \mathrm{mTOR}$ & N/A \\
\hline & SGI-1776 & PIM 1/2/3 kinase & $\mathrm{N} / \mathrm{A}$ \\
\hline & Volasertib & PLK 1/2/3 & N/A \\
\hline & UNC0642 & G9a/GLP & $\mathrm{N} / \mathrm{A}$ \\
\hline & prima1 & TP53 & N/A \\
\hline & AZD 8931 & EGFR, HER 2/3 & $\mathrm{N} / \mathrm{A}$ \\
\hline & JIB4 & JMJD & $\mathrm{N} / \mathrm{A}$ \\
\hline
\end{tabular}

tumor cells in vitro. BKM120 activity suggests that tumor growth and progression may be dependent on multiple pathways in PFN ARMS.

Additionally, agents associated with alteration of TP53 expression also demonstrated in vitro efficacy, including prima-1 $\left(I C_{50}=135 \mathrm{nM}\right)$ and CUDC-305 $\left(\mathrm{IC}_{50}=137 \mathrm{nM}, \mathrm{HSP} 90\right.$ inhibitor). Previous studies have found that prima-1 reactivates TP53, which induces high levels of apoptosis, and has demonstrated tumor reduction with no apparent toxicity in p53 mutant tumor xenografts in SCID mice (Bykov et al. 2002). Despite efforts in elucidating the mechanism of TP53 reactivation by prima-1, the mechanism remains largely unknown. Several factors, including hypoxic conditions and proteins present within and surrounding the cell, can affect the sensitivity of cancer cells to prima-1 (Rieber and Strasberg-Rieber 2012; Peng et al. 2013). Similarly, TP53 expression can be altered by HSP90 inhibitors (such as CUDC-305) and thus could potentially be beneficial for TP53 mutant patients (Lin et al. 2008).

\section{DISCUSSION}

In this study, we have presented a case of an 11-yr-old male with fusion negative ARMS bearing germline TP53 mutations, one of which (c.514G > T, p.Val172Phe) is a hereditary mutation likely predisposing to LFS. The index case patient presented with a tender left upper arm 
mass and no injury was initially noted. Tissue injury is widely reported to be a preceding factor in sarcoma development, with $13 \%$ of patients with accidental injury to the extremities at risk of developing sarcoma (Olsson and Wagner 2017), likely as a result of activation of satellite cells to generate muscle precursor cells to repair the skeletal muscle (Shadrach and Wagers 2011). We suspect that the prior supracondylar fracture of the distal humerus was a preceding factor that potentially promoted sarcoma development in conjunction with germline TP53 mutations.

Our genomic findings were consistent with previous genetic findings on PFN ARMS cases, including overexpression of FGFR4, CCND2, and IGF2 and significantly higher rates of nonsynonymous somatic mutations than the PAX-gene fusion positive tumors (PFP) (17.8 vs. 6.4, $P=0.0002$ ) (Shern et al. 2014). Whole-exome sequencing identified 109 nonsynonymous mutations for this case ( 3.6 mutations/MB), most consistent with a PFN genotype diagnosed at a later age. No somatic mutations in genes commonly mutated in PFN tumors were found in this case, including TP53, HRAS, KRAS, NRAS, FGFR4, PIK3CA, NF1, FBXW7, and BCOR (Stratton et al. 1989; Taylor et al. 2000, 2009; Shern et al. 2014). However, missense mutations were found in both TP53 and FGFR4 in the germline, as well as a nonsense-mediated decay mutation in NF1.

The biological significance of the FGFR4 germline variants and their contribution to sarcoma initiation, growth, and progression are largely unknown. Several previous studies have focused on the role of FGFR4 in RMS as a regulator of myogenic differentiation and muscle regeneration after injury. Somatic mutations in FGFR4 have been shown to contribute to the growth and metastasis of RMS, higher expression of FGFR4 correlates with poor survival, and FGFR4 suppresses phospho-Akt in RMS (Taylor et al. 2009). Germline variants in FGFR4 may play a similar role in promoting the invasion and metastasis of RMS.

The index case patient was treated with several targeted therapies, including temsirolimus (mTOR inhibitor), prexasertib (CHEK1 inhibitor), MK01775 (WEE1 inhibitor), pazopanib (multikinase inhibitor specific for VEGFR, PDGFR, c-KIT and FGFR), vorinostat (HDAC inhibitor), and palbociclib (CDK4/6 inhibitor). Functional testing of same or similar agents resulted in mixed concordance between in vitro response and clinical response. Patient tumor cells were highly sensitive to MK-1775, whereas clinical use resulted in continued disease progression. Efficacy of WEE1 inhibition may have differed from the cells that were harvested from intrapleural fluid because of host factors (e.g., drug metabolism) (HogenEsch and Nikitin 2012). HDAC inhibitor vorinostat was also provided to the patient with limited response, although multiple HDAC inhibitors (panobinostat, CUDC-907, and entinostat) were sensitive in vitro, whereas one HDAC inhibitor (CUDC-101) was not sensitive in vitro. CHEK1 inhibitor prexasertib was also used to treat the patient but did not suppress the patient's tumor growth, reflecting in vitro response of prexasertib. mTOR inhibitor temsirolimus (mTOR inhibitor) plus vinorelbine and cyclophosphamide stabilized the patient's disease. Two mTOR-associated agents (INK128 and BKM120) caused reduction in cell viability in vitro, whereas one mTOR agent (sirolimus) had limited in vitro efficacy.

TP53 interacting agents (prima- 1 directly and CUDC-305 operating through HSP90) were also promising in vitro. Treatment with prima-1 refolds TP53 in mutated cells into the wildtype conformation and restores sequence-specific DNA binding (Zhang et al. 2018). Other therapies that restore TP53 function, such as reactivating p53 and inducing tumor apoptosis (RITA), may merit investigation for PFN ARMS. Additionally, repression of TP53 is thought to be controlled by the TP53-p21-DREAM-E2F/CHR pathway, which regulates FAM83D (Engeland 2018). Future studies should aim to further understand how germline variants in FAM83D coupled with TP53 germline mutations contribute to sarcoma initiation, growth, and progression. Understanding the biological and clinical significance of FAM83D could have predictive and prognostic implications across several cancer types and may be relevant for the index case patient, given FAM83D aberrations present in patient's germline. 
COLD SPRING HARBOR Molecular Case Studies
Fusion-negative alveolar rhabdomyosarcoma
In summary, we have reported a PFN ARMS case with an inherited likely pathogenic germline TP53 mutation. The patient was resistant to several targeted therapies, demonstrating a need for improving our understanding of the etiology and pathogenesis of PFN ARMS and discovering effective therapeutic approaches with limited treatment-related toxicities. In this case study, several novel mutations were reported and potential therapeutic interventions were identified for further study to motivate preclinical and clinical studies that aim to improve survival for fusion-negative RMS.

\section{METHODS}

\section{Primary Cell Culture}

Approximately $2 \mathrm{~L}$ of pleural fluid was collected from a chest drain catheter and immediately chilled on ice. Twenty-four hours after initial collection, the fluid was transferred to 50-mL conical vials and centrifuged at $1200 \mathrm{rpm}$ for $10 \mathrm{~min}$. Resulting cell pellets were broken up with Gibco RPMI 1640 media and checked for viability with $0.4 \%$ trypan blue stain, then counted on a Life Technologies Countess II cell counter. Three million viable cells were used for immediate drug screening. Remaining cells were added to $15-\mathrm{cm}$ tissue culture plates containing Gibco RPMI 1640 media supplemented with 10\% fetal bovine serum and $1 \%$ penicillin/streptomycin. The plates were placed in a water-jacketed $37^{\circ} \mathrm{C}$ incubator with $5 \% \mathrm{CO}_{2}$. After $72 \mathrm{~h}$, the media was changed and the remaining cellular debris was removed. The semiadherent cell culture continued to expand for a minimum of 18 passages. Every two to five passages, stocks were collected in cryovials containing an FBS/10\% DMSO cryopreservation media and then slow cooled and stored in liquid nitrogen for future use.

\section{Drug Screen}

Cells from culture were counted, resuspended in media, and plated into white-walled 384well plates containing prediluted known concentrations of various drugs. After $72 \mathrm{~h}$, an equal volume of Cell-Titer Glo 2.0 (G9243, Promega) was added to each well. The cells were then incubated at room temperature while rocking in the dark for $15 \mathrm{~min}$. Cell viability was measured by luminescence with the BioTek Synergy HT plate reader (BioTek). EC 50 values were calculated using GraphPad Prism.

\section{Whole-Exome Sequencing}

Tumor and matched normal exome sequencing data were analyzed for the presence of somatic point mutation, somatic functional and structural mutations, potential germline mutations, polynucleotide insertions and deletions, and gene copy-number variation. Somatic mutations, variations, and indels were called using Genome Analysis Toolkit (GATK) Version 4.0 with strict calling criterion (Tumor logarithm, of odd [TLOD] scores above 6.3) with the GRCh38 human reference genome. Gene copy-number variations were identified using SAMtools and VarScan2 quantified as a log ratio of tumor copy to normal copy. Regions with a log ratio $>0.40$ were called as gained, regions with a log ratio $<-0.40$ were called as lost. Genes overlapping gained or lost regions by at least $15 \%$ of the gene's genomic region were called as gained or lost, respectively. Sequencing coverage is provided in Supplemental Table 5.

\section{RNA Sequencing}

RNA sequencing data was analyzed for gene expression and gene fusion events. Transcriptome data was aligned to STAR-derived human transcriptome from GRCh38 human reference genome. Normalized gene expression was quantified using RSEM. Nontumor 
gene expression data was not provided, thus region-specific, skeletal muscle tissue, gene expression data was accessed from the GTEx project to serve as a population normal and to identify under expressed and overexpressed genes. Gene fusion events were identified using STAR-Fusion (Haas et al. 2019) and FusionCatcher (Nicorici et al. 2014) to identify transcriptome reads around the junction of fused genes and identify probable gene fusion events. Both fusion analyses were also performed using the GRCh38 human reference genome. Sequencing coverage is provided in Supplemental Table 5.

\section{Hierarchical Clustering}

The hierarchical clustering analysis approach was published previously (Ricker et al. 2020). Briefly, CF-00449 sequencing data was clustered among a cohort of RMS samples collected from multiple sources. Unsupervised hierarchical clustering was performed using averagelinkage clustering and Euclidean distance metric. Hierarchical clustering analysis was performed in RStudio Version 3.6.2.

\section{Circos Plots}

Circos plots to visualize the collection of genomics and transcriptomics data were generated using tumor DNA exome, normal DNA exome, and tumor RNA sequencing data. Genes are identified as bearing variants, having increased gene copy-number, and being overexpressed. Also identified in blue are genes involved in identified gene fusion events, and genes identified as being of interest because of overarching genetic and transcriptomic features in the tumor samples. (See Fig. 3.)

\section{ADDITIONAL INFORMATION}

\section{Data Deposition and Access}

All raw data was deposited to our CuReFAST database. DNA and RNA sequencing data from the patient's sample are available through the European Genome-Phenome Archive (EGA) under accession ID EGAS00001004828. For hierarchical clustering analysis, data from the Gene Expression Omnibus (GSM758578, GSE138269, GSM984615), EGA (EGAS00001003981), and dbGaP (accession number phs001121.v1.p1) were used. Champions Oncology data were received from the Champions TumorGraft database. Genomics data from PDX models from The Jackson Laboratory are available through the Mouse Tumor Biology Database (http://tumor.informatics.jax.org/mtbwi/pdxSearch.do).

\section{Ethics Statement}

The genetic studies and publication of clinical details was approved by the Children's Cancer Therapy Development Institute Institutional Review Board (IRB) and with full written informed consent obtained from parents of the proband.

\section{Acknowledgments}

We are tremendously grateful for all the patients who kindly donated their tumor tissue as

Competing Interest Statement

The authors have declared no competing interest.

Received November 1, 2020; accepted in revised form January 11, 2021. part of our CuReFAST initiative.

\section{Author Contributions}

C.K. and C.A.R. designed the study. C.K., C.A.R., A.W., A.M., E.R.R., M.L., and N.E.B. collected and analyzed the data. G.S., R.G.I., and W.S. contributed materials. C.K., C.A.R., A.D.W., and N.E.B. wrote the manuscript. 


\section{Funding}

This work was supported through Building Blocks to a Cure (1:1 match) crowdfunding campaign on Consano (https://consano.org/projects/building-blocks-to-a-cure-11-match/), the Children's Cancer Project, and the Sam Day Foundation.

\section{REFERENCES}

Ballinger ML, Mitchell G, Thomas DM. 2015. Surveillance recommendations for patients with germline TP53 mutations. Curr Opin Oncol 27: 332-337. doi:10.1097/CCO.0000000000000200

Bharathy N, Berlow NE, Wang E, Abraham J, Settelmeyer TP, Hooper JE, Svalina MN, Ishikawa Y, Zientek K, Bajwa Z, et al. 2018. The HDAC3-SMARCA4-miR-27a axis promotes expression of the PAX3:FOXO1 fusion oncogene in rhabdomyosarcoma. Sci Signal 11: eaau7632. doi:10.1126/scisignal.aau7632

Bolkestein M, Wong JKL, Thewes V, Körber V, Hlevnjak M, Elgaafary S, Schulze M, Kommoss FKF, Sinn H-P, Anzeneder T, et al. 2020. Chromothripsis in human breast cancer. Cancer Res 80: 4918-4931. doi:10.1158/ 0008-5472.CAN-20-1920

Breneman JC, Lyden E, Pappo AS, Link MP, Anderson JR, Parham DM, Qualman SJ, Wharam MD, Donaldson SS, Maurer HM, et al. 2003. Prognostic factors and clinical outcomes in children and adolescents with metastatic rhabdomyosarcoma-a report from the Intergroup Rhabdomyosarcoma Study IV. J Clin Oncol 21: 78-84. doi:10.1200/JCO.2003.06.129

Bykov VJ, Issaeva N, Shilov A, Hultcrantz M, Pugacheva E, Chumakov P, Bergman J, Wiman KG, Selivanova G. 2002. Restoration of the tumor suppressor function to mutant p53 by a low-molecular-weight compound. Nat Med 8: 282-288. doi:10.1038/nm0302-282

Casey DL, Wexler LH, Pitter KL, Samstein RM, Slotkin EK, Wolden SL. 2020. Genomic determinants of clinical outcomes in rhabdomyosarcoma. Clin Cancer Res 26: 1135-1140. doi:10.1158/1078-0432.CCR19-2631

Consortium GT. 2013. The Genotype-Tissue Expression (GTEx) project. Nat Genet 45: 580-585. doi:10.1038/ ng. 2653

Cortés-Ciriano I, Lee JJ-K, Xi R, Jain D, Jung YL, Yang L, Gordenin D, Klimczak LJ, Zhang C-Z, Pellman DS, et al. 2020. Comprehensive analysis of chromothripsis in 2,658 human cancers using whole-genome sequencing. Nat Genet 52: 331-341. doi:10.1038/s41588-019-0576-7

Crist WM, Anderson JR, Meza JL, Fryer C, Raney RB, Ruymann FB, Breneman J, Qualman SJ, Wiener E, Wharam M, et al. 2001. Intergroup rhabdomyosarcoma study-IV: results for patients with nonmetastatic disease. J Clin Oncol 19: 3091-3102. doi:10.1200/JCO.2001.19.12.3091

Davis LE, Keller C. 2012. Integrative biology of rhabdomyosarcoma using genetic murine models. AACR 2012 Annual Meeting Educational Book.

Engeland K. 2018. Cell cycle arrest through indirect transcriptional repression by p53: I have a DREAM. Cell Death Differ 25: 114-132. doi:10.1038/cdd.2017.172

Farid M, Ngeow J. 2016. Sarcomas associated with genetic cancer predisposition syndromes: a review. Oncologist 21: 1002-1013. doi:10.1634/theoncologist.2016-0079

Gonzalez KD, Noltner KA, Buzin CH, Gu D, Wen-Fong CY, Nguyen VQ, Han JH, Lowstuter K, Longmate J, Sommer SS, et al. 2009. Beyond Li-Fraumeni syndrome: clinical characteristics of families with p53 germline mutations. J Clin Oncol 27: 1250-1256. doi:10.1200/JCO.2008.16.6959

Haas BJ, Dobin A, Li B, Stransky N, Pochet N, Regev A. 2019. Accuracy assessment of fusion transcript detection via read-mapping and de novo fusion transcript assembly-based methods. Genome Biol 20: 213. doi:10.1186/s13059-019-1842-9

Hainaut P, Soussi T, Shomer B, Hollstein M, Greenblatt M, Hovig E, Harris CC, Montesano R. 1997. Database of p53 gene somatic mutations in human tumors and cell lines: updated compilation and future prospects. Nucleic Acids Res 25: 151-157. doi:10.1093/nar/25.1.151

HogenEsch H, Nikitin AY. 2012. Challenges in pre-clinical testing of anti-cancer drugs in cell culture and in animal models. J Control Release 164: 183-186. doi:10.1016/j.jconrel.2012.02.031

Inamura K, Shimoji T, Ninomiya H, Hiramatsu M, Okui M, Satoh Y, Okumura S, Nakagawa K, Noda T, Fukayama $M$, et al. 2007. A metastatic signature in entire lung adenocarcinomas irrespective of morphological heterogeneity. Hum Pathol 38: 702-709. doi:10.1016/j.humpath.2006.11.019

Li Y, Seto E. 2016. HDACs and HDAC inhibitors in cancer development and therapy. Cold Spring Harb Perspect Med 6: a026831. doi:10.1101/cshperspect.a026831

Liao W, Liu W, Liu X, Yuan Q, Ou Y, Qi Y, Huang W, Wang Y, Huang J. 2015. Upregulation of FAM83D affects the proliferation and invasion of hepatocellular carcinoma. Oncotarget 6: 24132-24147. doi:10.18632/ oncotarget.4432 
Lin K, Rockliffe N, Johnson GG, Sherrington PD, Pettitt AR. 2008. Hsp90 inhibition has opposing effects on wild-type and mutant p53 and induces p21 expression and cytotoxicity irrespective of p53/ ATM status in chronic lymphocytic leukaemia cells. Oncogene 27: 2445-2455. doi:10.1038/sj.onc .1210893

Malempati S, Hawkins DS. 2012. Rhabdomyosarcoma: review of the Children's Oncology Group (COG) SoftTissue Sarcoma Committee experience and rationale for current COG studies. Pediatr Blood Cancer 59: 510. doi:10.1002/pbc. 24118

Nicorici D, Satalan M, Edgren H, Kangaspeska S, Murumagi A, Kallioniemi O, Virtanen S, Kilkku O. 2014. FusionCatcher-a tool for finding somatic fusion genes in paired-end RNA-sequencing data. bioRxiv doi:10.1101/011650

Ognjanovic S, Olivier M, Bergemann TL, Hainaut P. 2012. Sarcomas in TP53 germline mutation carriers: a review of the IARC TP53 database. Cancer 118: 1387-1396. doi:10.1002/cncr.26390

Olivier M, Goldgar DE, Sodha N, Ohgaki H, Kleihues P, Hainaut P, Eeles RA. 2003. Li-Fraumeni and related syndromes: correlation between tumor type, family structure, and TP53 genotype. Cancer Res 63: 6643-6650.

Olsson HL, Wagner P. 2017. Tissue trauma and the subsequent risk of soft tissue sarcoma of the extremity: a population based case-control study in Sweden. J Clin Oncol 35: e13088. doi:10.1200/JCO.2017.35 .15_suppl.e13088

Palmero El, Achatz MI, Ashton-Prolla P, Olivier M, Hainaut P. 2010. Tumor protein 53 mutations and inherited cancer: beyond Li-Fraumeni syndrome. Curr Opin Oncol 22: 64-69. doi:10.1097/CCO.0b013e328 $333 b f 00$

Peng X, Zhang MQ, Conserva F, Hosny G, Selivanova G, Bykov VJ, Arner ES, Wiman KG. 2013. APR-246/ PRIMA-1 ${ }^{\mathrm{MET}}$ inhibits thioredoxin reductase 1 and converts the enzyme to a dedicated NADPH oxidase. Cell Death Dis 4: e881. doi:10.1038/cddis.2013.417

Pondrom M, Bougeard G, Karanian M, Bonneau-Lagacherie J, Boulanger C, Boutroux H, Briandet C, Chevreau C, Corradini N, Coze C, et al. 2020. Rhabdomyosarcoma associated with germline TP53 alteration in children and adolescents: the French experience. Pediatr Blood Cancer 67: e28486.

Ramakrishna M, Williams LH, Boyle SE, Bearfoot JL, Sridhar A, Speed TP, Gorringe KL, Campbell IG. 2010. Identification of candidate growth promoting genes in ovarian cancer through integrated copy number and expression analysis. PLoS ONE 5: e9983. doi:10.1371/journal.pone.0009983

Ricker CA, Crawford K, Matlock K, Lathara M, Seguin B, Rudzinski ER, Berlow NE, Keller C. 2020. Defining an embryonal rhabdomyosarcoma endotype. Cold Spring Harb Mol Case Stud 6: a005066. doi:10.1101/mcs .005066

Rieber M, Strasberg-Rieber M. 2012. Hypoxia, Mn-SOD and H(2)O(2) regulate p53 reactivation and PRIMA-1 toxicity irrespective of p53 status in human breast cancer cells. Biochem Pharmacol 84: 1563-1570. doi:10 .1016/j.bcp.2012.09.003

Rudzinski ER, Anderson JR, Chi YY, Gastier-Foster JM, Astbury C, Barr FG, Skapek SX, Hawkins DS, Weigel BJ, Pappo A, et al. 2017. Histology, fusion status, and outcome in metastatic rhabdomyosarcoma: a report from the Children's Oncology Group. Pediatr Blood Cancer 64: 10.1002/pbc.26645. doi:10.1002/pbc .26645

Shadrach JL, Wagers AJ. 2011. Stem cells for skeletal muscle repair. Philos Trans R Soc Lond B Biol Sci 366: 2297-2306. doi:10.1098/rstb.2011.0027

Shern JF, Chen L, Chmielecki J, Wei JS, Patidar R, Rosenberg M, Ambrogio L, Auclair D, Wang J, Song YK, et al. 2014. Comprehensive genomic analysis of rhabdomyosarcoma reveals a landscape of alterations affecting a common genetic axis in fusion-positive and fusion-negative tumors. Cancer Discov 4: 216-231. doi:10.1158/2159-8290.CD-13-0639

Sherry ST, Ward MH, Kholodov M, Baker J, Phan L, Smigielski EM, Sirotkin K. 2001. dbSNP: the NCBI database of genetic variation. Nucleic Acids Res 29: 308-311. doi:10.1093/nar/29.1.308

Stratton MR, Fisher C, Gusterson BA, Cooper CS. 1989. Detection of point mutations in N-ras and K-ras genes of human embryonal rhabdomyosarcomas using oligonucleotide probes and the polymerase chain reaction. Cancer Res 49: 6324-6327.

Taylor AC, Shu L, Danks MK, Poquette CA, Shetty S, Thayer MJ, Houghton PJ, Harris LC. 2000. P53 mutation and MDM2 amplification frequency in pediatric rhabdomyosarcoma tumors and cell lines. Med Pediatr Oncol 35: 96-103. doi:10.1002/1096-911X(200008)35:2<96::AID-MPO2>3.0.CO ;2-Z

Taylor JG, Cheuk AT, Tsang PS, Chung JY, Song YK, Desai K, Yu Y, Chen OR, Shah K, Youngblood V, et al 2009. Identification of FGFR4-activating mutations in human rhabdomyosarcomas that promote metastasis in xenotransplanted models. J Clin Invest 119: 3395-3407.

Walian PJ, Hang B, Mao JH. 2016. Prognostic significance of FAM83D gene expression across human cancer types. Oncotarget 7: 3332-3340. doi:10.18632/oncotarget.6620 
Wang Z, Liu Y, Zhang P, Zhang W, Wang W, Curr K, Wei G, Mao JH. 2013. FAM83D promotes cell proliferation and motility by downregulating tumor suppressor gene FBXW7. Oncotarget 4: 2476-2486. doi:10.18632/ oncotarget.1581

Wang D, Han S, Peng R, Wang X, Yang XX, Yang RJ, Jiao CY, Ding D, Ji GW, Li XC. 2015. FAM83D activates the MEK/ERK signaling pathway and promotes cell proliferation in hepatocellular carcinoma. Biochem Biophys Res Commun 458: 313-320. doi:10.1016/j.bbrc.2015.01.108

Williams BA, Williams KM, Doyle J, Stephens D, Greenberg M, Malkin D, Pappo AS. 2004. Metastatic rhabdomyosarcoma: a retrospective review of patients treated at the hospital for sick children between 1989 and 1999. J Pediatr Hematol Oncol 26: 243-247. doi:10.1097/00043426-200404000-00006

Williamson D, Missiaglia E, de Reyniès A, Pierron G, Thuille B, Palenzuela G, Thway K, Orbach D, Laé M, Fréneaux $P$, et al. 2010. Fusion gene-negative alveolar rhabdomyosarcoma is clinically and molecularly indistinguishable from embryonal rhabdomyosarcoma. J Clin Oncol 28: 2151-2158. doi:10.1200/JCO.2009 .26 .3814

Zhang Q, Bykov VJN, Wiman KG, Zawacka-Pankau J. 2018. APR-246 reactivates mutant p53 by targeting cysteines 124 and 277. Cell Death Dis 9: 439. doi:10.1038/s41419-018-0463-7 


\section{COLD SPRING HARBOR Molecular Case Studies}

\section{Refractory alveolar rhabdomyosarcoma in an 11-year-old male}

Cora A. Ricker, Andrew D. Woods, William Simonson, et al.

Cold Spring Harb Mol Case Stud 2021, 7: a005983 originally published online January 12, 2021

Access the most recent version at doi: $10.1101 /$ mcs.a005983

\section{Supplementary http://molecularcasestudies.cshlp.org/content/suppl/2021/01/14/mcs.a005983.D} Material C1

References This article cites 43 articles, 14 of which can be accessed free at: http://molecularcasestudies.cshlp.org/content/7/1/a005983.full.html\#ref-list-1

License This article is distributed under the terms of the Creative Commons

Attribution-NonCommercial License, which permits reuse and redistribution, except for commercial purposes, provided that the original author and source are credited.

Email Alerting Receive free email alerts when new articles cite this article - sign up in the box at the Service top right corner of the article or click here. 